\title{
Putative invasive pulmonary aspergillosis in apparently immunocompetent patients within medical wards and intensive care units
}

\author{
Babatunde Edun $^{1} \cdot$ Mark Alan Tidswell $^{1}$
}

Received: 28 April 2021 / Accepted: 30 April 2021 / Published online: 17 May 2021

c) Società Italiana di Medicina Interna (SIMI) 2021

Despite advances in evaluation and tests, patients in the ICU may have a suspected infection without a specific microbiological diagnosis. Candida and Aspergillus infections are the most frequent fungal infections in ICU patients, and aspergillosis carries a high mortality. Invasive pulmonary aspergillosis (IPA) is recognized as a cause of pneumonia in immunocompromised patients, and EORTC/MSG guidelines provide diagnostic criteria based on biopsy or culture from a sterile site, or (1) lower respiratory tract specimen (2) galactomannan antigen in plasma and/or alveolar lavage fluid, and other clinical features [1]. In contrast, for patients who are not immune compromised, there is a lack of specific diagnostic testing for IPA [2]. Critically ill patients, especially those with sepsis, may be relatively immune suppressed even if they do not have a primary diagnosis traditionally associated with immune compromise. Sepsis can cause immune suppression characterized by lymphopenia with increased susceptibility to secondary infection and mortality [3]. Typically, these patients cannot tolerate a lung biopsy or invasive tests to achieve a diagnosis of proven IPA [2]. Furthermore, even the terms "probable" or "possible" invasive pulmonary aspergillosis were developed for classical immune-compromised hosts and may not be applicable to the ICU patient [1]. However, given the case fatality rate of over $50 \%$ associated with IPA, it is important to have tools to establish a diagnosis [4].

An alternate diagnostic algorithm for IPA in the nonimmune-compromised critically ill patient who may not fit EORTC/MSG criteria was evaluated by Blot and colleagues in 2012. This clinical algorithm, studied in 524 critically ill patients, made use of the EORTC/MSG criteria for proven invasive pulmonary aspergillosis, and added a category

Mark Alan Tidswell

mark.tidswell@baystatehealth.org

1 Pulmonary and Critical Care Division, Baystate Medical Center, Springfield, MA 01199, USA termed putative invasive pulmonary aspergillosis (PIPA), which first requires a positive lower respiratory tract culture and incorporates additional clinical and host criteria. Under these guidelines, patients who did not fit into either the proven or putative category were defined as being colonized. When compared with histopathology-controlled patients, this algorithm was found to be $92 \%$ sensitive and $61 \%$ specific [2].

In this issue of IAEM, Corcione and colleagues [5] used these same criteria for PIPA [2] to perform a retrospective observational single-center study of all hospitalized patients with a positive Aspergillus culture from a lower airway sample obtained via bronchoalveolar lavage (BAL) or bronchial aspirate (BA). The authors applied the defined clinical, host, and imaging criteria to establish a diagnosis of PIPA. Those patients who did not meet criteria were classified as colonized.

Of the more than 80,000 patients presenting during a 4-year period, 76 had a positive lower respiratory tract culture, of whom 52 met criteria for a diagnosis of PIPA (68\%) and the remaining 24 were said to be colonized. The predominant Aspergillus species was A. fumigatus in $73 \%$ of isolates. The analysis included patients on the medical wards and in the ICU. Of the 58 ICU patients in this study, 36 met the criteria for PIPA. Aspergillus positive lower airway culture results occurred at a rate of 4.94 per thousand patients in ICU and 0.28 per thousand patients in the medical wards. The authors add to the medical literature by reporting a rate for medical ward patients, and their results are similar to reported rates for ICU patients.

Some in the study population were immune compromised as defined by EORTC/MSG. For instance, 16 patients were on immune-suppressive medication. Chronic diseases such as cardiovascular, obstructive airways, and malignancy were frequent in the population. ICU patients had sepsis, required supportive therapies, and the majority had abdominal or trauma surgery. A multivariate analysis found a high odds 
ratio of PIPA associated with steroid therapy, or absence of antifungal therapy, among other factors-suggesting a potential role for earlier diagnosis and treatment. In light of the small sample size and many comparisons, the results are not conclusive, but do suggest important questions for further study.

Of the 76 patients, there were 37 survivors, and mortality at 21 days was $51 \%$. This is similar to mortality rates in other studies of PIPA. Among patients with PIPA that died $82 \%$ received antifungal therapy. While one might expect treatment to confer a survival benefit this does not appear to be the case, suggesting that the timing of treatment relative to disease course or other comorbidities contributed to mortality [6]. Similarly, in the study by Blot et al., of the 524 subjects with positive endotracheal aspirate cultures, 199 (38\%) met criteria for a diagnosis of PIPA, and mortality in the PIPA group was $67.5 \%$ despite $91 \%$ receiving antifungal therapy [2]. Might earlier identification and treatment with antifungal therapy improve outcome? Are these very ill subjects who would die from underlying disease despite treatment for PIPA? The authors note as an important study limitation that severity of illness scores such as APACHE were not available in this retrospective analysis.

Also, in Corcione's paper, none of the 76 subjects with a positive lower respiratory culture was diagnosed with proven IPA. The authors do not state how many subjects underwent a lung biopsy, and this highlights a drawback of using a positive respiratory tract culture as the only entry criterion for the diagnosis of invasive pulmonary Aspergillosis. There may have been additional patients with PIPA. Cultures of BAL and BA are not entirely reliable and are known to have highly variable sensitivity-as low as $30 \%$ in certain settings [6].

Indeed, criteria used to make the diagnosis of IPA in ICU patients may underestimate the actual rate. In the Blot et al. study, 24\% patients in the subset of histopathology-controlled cases were diagnosed with proven IPA despite having a negative respiratory culture. That study compared the use of clinical criteria to histopathologic data and found that clinical criteria were only $92 \%$ sensitive at detecting cases of IPA, likely due to reliance on the requirement for positive culture along with clinical and radiologic factors [1]. Using a diagnostic approach that did not require a positive Aspergillus culture, Loughlin, et al. identified a high rate of probable Aspergillus infected patients for 24 (12\%) of 194 nonneutropenic critically ill adults with worsening alveolar infiltrates, fever, purulent secretions, and one or more of the following: positive galactomannan index in BAL fluid or serum, positive BAL fluid culture, or positive histology [7]. Hage and colleagues showed that serum and/or BAL galactomannan was up to $96 \%$ sensitive and, along with other criteria, was enough to make a diagnosis of probable IPA in the immune compromised without waiting for culture [8]. A similar trend was seen in the 2012 paper by Blot et al. which reported that $45 \%$ of patients had EORTC host factors. These subjects might have been classified as probable IPA using EORTC/ MSG criteria which allow non-microbiological tests such as serum/BAL galactomannan, and Aspergillus PCR without the need for a positive lower respiratory tract culture.

To address the issue of invasive aspergillosis in critically ill non-immune-compromised hosts, the EORTC/MSG ICU Working Group in 2019 revised their definition of possible invasive aspergillosis. Patient criteria were expanded to include chronic obstructive pulmonary disease (COPD), liver cirrhosis, influenza pneumonia, and COVID-19 pneumonia. They also included absolute cut-off values for a positive BAL or serum galactomannan [9]. However, they were unable to generate specific recommendations regarding the ICU population for multiple reasons including significant heterogeneity in pre-existing conditions and heterogeneity in radiologic findings in the ICU patient [10]. Given these limitations, the PIPA clinical algorithm remains a useful diagnostic tool for invasive aspergillosis in the ICU population.

Cases of invasive aspergillosis co-infection have now been reported in mechanically ventilated patients with pneumonia due to SARS-COV2, and termed COVID-19 associated pulmonary aspergillosis (CAPA), at rates as high as $14-27 \%$ in some populations [11, 12]. Obtaining a lung biopsy to prove IPA would pose significant risk to both patient and staff. The work by Corcione and colleagues is a timely reminder of the utility of a clinical algorithm created for the ICU population [13]. Although IPA is infrequent, prompt consideration of Aspergillus is important in the evaluation of undiagnosed pulmonary infiltrates in ICU patients.

\section{Declarations}

Conflict of interest Dr. Edun has no conflicts of interest relevant to this article. Dr. Tidswell has no conflicts of interest relevant to this article.

Human and animal rights statement This commentary is the original work of the named authors, who reviewed previously published information. No new human or animal studies were performed by the authors.

Informed consent For this type of study no informed consent is required.

\section{References}

1. Bassetti M, Azoulay E, Kullberg BJ et al (2021) EORTC/ MSGERC definitions of invasive fungal diseases: summary of activities of the Intensive Care Unit Working Group. Clin Infect Dis 72(Suppl 2):S121-s127 
2. Blot SI, Taccone FS, Van den Abeele AM et al (2012) A clinical algorithm to diagnose invasive pulmonary aspergillosis in critically ill patients. Am J Respir Crit Care Med 186(1):56-64

3. Hotchkiss RS, Monneret G, Payen D (2013) Immunosuppression in sepsis: a novel understanding of the disorder and a new therapeutic approach. Lancet Infect Dis 13(3):260-268

4. Lin S-J, Schranz J, Teutsch SM (2001) Aspergillosis casefatality rate: systematic review of the literature. Clin Infect Dis 32(3):358-366

5. Corcione S, Lupia T, Raviolo S et al (2021) Putative invasive pulmonary aspergillosis within medical wards and intensive care units: a 4-year retrospective, observational, single-centre study. Intern Emerg Med. https://doi.org/10.1007/s11739-021-02705-z

6. Delsuc C, Cottereau A, Frealle E et al (2015) Putative invasive pulmonary aspergillosis in critically ill patients with chronic obstructive pulmonary disease: a matched cohort study. Crit Care 19:421

7. Loughlin L, Hellyer TP, TP, White PL, et al (2020) Pulmonary aspergillosis in patients with suspected ventilator-associated pneumonia in UK ICUs. Am J Respir Crit Care Med 202(8):1125-1132

8. Hage CA, Carmona EM, Epelbaum O et al (2019) Microbiological laboratory testing in the diagnosis of fungal infections in pulmonary and critical care practice an official american thoracic society clinical practice guideline. Am J Respir Crit Care Med 200(5):535-550

9. Donnelly JP, Chen SC, Kauffman CA et al (2020) Revision and update of the consensus definitions of invasive fungal disease from the european organization for research and treatment of cancer and the mycoses study group education and research consortium. Clin Infect Dis 71(6):1367-1376

10. Bassetti M, Scudeller L, Giacobbe DR et al (2019) Developing definitions for invasive fungal diseases in critically ill adult patients in intensive care units. Protocol of the FUNgal infections Definitions in ICU patients (FUNDICU) project. Mycoses 62(4):310-319

11. White PL, Dhillon R, Cordey A et al (2020) A national strategy to diagnose COVID-19 associated invasive fungal disease in the ICU. Clin Infect Dis. https://doi.org/10.2139/ssrn.3644400

12. Bartoletti M, Pascale R, Cricca M et al (2020) PREDICO study group. Epidemiology of invasive pulmonary aspergillosis among COVID-19 intubated patients: a prospective study. Clin Infect Dis. https://doi.org/10.1093/cid/ciaa1065

13. Koehler P, Bassetti M, Chakrabarti A et al (2020) Defining and managing COVID-19-associated pulmonary aspergillosis: the 2020 ECMM/ISHAM consensus criteria for research and clinical guidance. Lancet Infect Dis. https://doi.org/10.1016/S14733099(20)30847-1

Publisher's Note Springer Nature remains neutral with regard to jurisdictional claims in published maps and institutional affiliations. 